\title{
PASOS RETÓRICOS EN TRABAJOS FINALES DE GRADO DE INGENIERÍA CIVIL
}

\author{
María Virginia Hael \\ CONICET \\ INVELEC
}

Resumen: El objetivo de este trabajo es descubrir si estudiantes de Ingeniería Civil de una universidad pública argentina incluyen los segmentos textuales (Gnutzmann y Oldenburg, 1991 y Ciapuscio y Otañi, 2002) y los pasos retóricos (Swales, 1990) prototípicos del artículo científico en sus trabajos finales de grado. Si bien no se trata de artículos de investigación, estos pasos retóricos también podían ser aplicados a las tesinas de licenciatura y a los trabajos finales de grado (Hael, 2010). Nos interesa estudiar este tema en virtud de descubrir qué papel juega la argumentación en este tipo de escritos, teniendo en cuenta que tanto los segmentos textuales como los pasos retóricos pueden dar cuenta de dos tipos de argumentación académica: la demostrativa y la dialéctica (Padilla, Douglas y Lopez, 2011).

Palabras clave: Pasos retóricos, segmentos textuales, trabajos finales de grado, argumentación académica, Ingeniería Civil.

\begin{abstract}
The main objective in this paper is to find out if Argentinean Public University Engineering students include textual parts (Gnutzmann \& Oldenburg, 1991 \& and Ciapuscio \& Otañi, 2002) and rhetoric steps (Swales, 1990) of scientific papers in their undergraduate final thesis. Even when we are speaking about paper parts and rhetoric steps, they could be thought for their final thesis too (Hael, 2010). We are interested in study this subject to find out if argumentation have place -and what place that have- in this writing works. Textual parts and rhetoric steps could show two tipes of academic argumentation: demonstrative argumentation and dialectical argumentation (Padilla, Douglas and Lopez, 2011).
\end{abstract}

Keywords: Rhetoric Steps, texts segments, graduation final writing work, academia argumentation, Engineering. 


\section{Introducción}

Este trabajo forma parte de una investigación doctoral en curso que aborda el modo en que los estudiantes de diferentes disciplinas académicas construyen argumentativamente el conocimiento en tesinas de licenciatura y trabajos finales de grado.

Para este trabajo en particular, nos interesó abordar los pasos retóricos que los estudiantes de Ingeniería Civil de una universidad pública argentina utilizan a la hora de construir sus trabajos finales de grado. Las partes canónicas de escritos académicos -Introducción, Metodología, Resultados, Discusión, Conclusión, Bibliografía- (Gnutzmann y Oldenburg, 1991 y Ciapuscio y Otañi, 2002) y los pasos retóricos (Swales, 1990) se vinculan con tres tipos de argumentación académica (Padilla et al, 2011): una argumentación demostrativa, que implica la relación entre el marco teórico, las hipótesis e interrogantes, los resultados y las conclusiones; una argumentación persuasiva que intenta comunicar los resultados de la investigación de la manera más clara posible, atendiendo al posible lector; y una argumentación dialéctica, que pone en relación los resultados de la propia investigación con resultados de otras investigaciones y que cobra más fuerza en el apartado "discusión". Es en virtud del quehacer argumentativo de los trabajos finales de grado de Ingeniería Civil que nos interesa especialmente estudiar las partes canónicas y los pasos retóricos en estos escritos, ya que consideramos que a través del análisis de sus segmentos textuales podremos descubrir qué cabida tienen la 
argumentación demostrativa y la argumentación dialéctica en estos escritos.

Cabe destacar, también, que se trata de un área de vacancia en los estudios sobre textos académicos: Padilla y Carlino (2010) consideran que si bien en los últimos tiempos se han realizado numerosos estudios sobre la escritura académica, no existen muchas investigaciones que establezcan un vínculo entre aquella y la argumentación. Es por esto que consideramos fundamental el abordaje de la escritura disciplinar, pero nucleado en la construcción argumentativa del saber científico. Asimismo, el género tesis de grado -en este caso particular, trabajos finales de grado- ha sido poco abordado en investigaciones precedentes, en contraposición con el extenso análisis que se ha hecho sobre las tesis de posgrado (Marinkovich y Salazar, 2011). Si bien existen investigaciones precedentes sobre alfabetizaciones académicas (Carlino, 2004, 2005, 2006, 2011 y 2013; Parodi, 2008; MottaRoth, 2001, 2009), argumentación en la universidad (Soto Vergara, 2002，2003，2004，2005，2004-2005; Soto Vergara y Zenteno, 2001-2003, 2004; Larrain, 2009), sobre escritura disciplinar (Arnoux, Alvarado, Balmayor, di Stéfano, Pereira y Silvestri, 1996; Arnoux y Alvarado, 1999; Arnoux, Di Stefano y Pereira, 2002; Marín y Hall, 2003; Vázquez y Miras, 2004, Marinkovich y Salazar, 2011; Velásquez Rivera y Córdoba Jiménez, 2011; Tapia y Marinkovich, 2013; Marinkovich y Córdova, 2014; Lea y Street, 1998; Hillard y Harris, 2003; Lillis y Turner, 2001) y sobre la función epistémica de la argumentación (Perin, Keselman y Monopoli, 2003; ZuberSkerritt y Perry, 2002; Wolfe, 2011), no hemos encontrado 
investigaciones que vinculen estas tres líneas en el ámbito universitario. Por este motivo, consideramos necesario realizar este estudio.

\section{Antecedentes}

Dentro del marco teórico general en el que se enmarca nuestro trabajo, y al que hemos hecho breve referencia en el apartado anterior, resultan de especial interés los estudios internacionales acerca de prácticas de lectura, escritura y argumentación en estudiantes universitarios de diferentes disciplinas (Perin, Keselman y Monopoli, 2003; Zuber-Skerritt y Perry, 2002; Wolfe, 2011). Estos estudios consideran que las tareas que suelen asignarse en la universidad requieren de un desarrollo argumentativo, y sostienen, a su vez, que tanto en cada carrera como en cada asignatura, los modos de argumentación solicitados son diferentes. Para nuestro trabajo, esto es fundamental: así como en relación con distintos trabajos y asignaturas la argumentación puede cobrar diferentes formas, consideramos que en distintas disciplinas, la argumentación también tendrá formas específicas de construcción del saber académico-científico, y es en virtud de esto que abordamos las prácticas argumentativas en relación con los segmentos textuales y los pasos retóricos de los trabajos finales de grado.

Uno de los antecedentes más relevantes en cuanto al estudio del género tesis de licenciatura -o trabajo final de grado- es la investigación de Tamola de Spiegel (2007) que, a partir del análisis de once tesinas de licenciatura de diferentes disciplinas, 
aborda el género desde la situación comunicativa, la estructura y los procedimientos de desarrollo textual. Describe al género tesina o tesis de grado como un texto escrito, en primera instancia, en el que se exponen los resultados de una investigación y que ha sido coordinado por un director. En algunas universidades, luego de la presentación por escrito, también se hace una exposición oral ante un tribunal compuesto por el director y otros dos profesores vinculados a la temática.

Tamola de Spiegel (2007) establece vínculos entre este género y la argumentación académica:

Los tesistas utilizan la lengua escrita no solo para informar sino para convencer con los argumentos acerca de los resultados de su estudio, a fin de conseguir la adhesión del oyente/lector, que, generalmente, es miembro de la comunidad de discurso en la que se inserta el trabajo (Tamola de Spiegel, 2007: 237)

A esto, agrega que transmitir información y convencer son dos funciones básicas e importantes de la tesis de licenciatura, en la que, además, se genera conocimiento novedoso. A partir de estas consideraciones, podemos establecer algunos vínculos con nuestro trabajo: la relación entre escribir, argumentar y generar conocimiento disciplinar es constitutiva del género al que nos abocamos.

Dentro de su descripción sobre los segmentos textuales y los procedimientos que están incluidos en ellos, destaca, en primer lugar, una introducción, que consta de una descripción general del tema abordado, en el que se reconoce una laguna o 
problema por resolver, la relevancia de estudiar ese tema, el planteamiento del problema, que se asocia a otras tres categorías: las preguntas de investigación, los objetivos y propósitos y la justificación. Además se presentan la revisión bibliográfica y un adelanto de lo que se tratará en cada capítulo. En segundo lugar, presenta el núcleo textual, en el que los tesistas elaboran su marco teórico y de antecedentes, revisan la bibliografía y construyen su perspectiva teórica. Es aquí también donde se presenta la metodología, en la que se incluyen el problema, las hipótesis, la especificación de las variables, los objetivos generales y específicos, el tipo de investigación y de diseño, las unidades de análisis, la descripción de la población y la muestra, las técnicas e instrumentos de recolección de los datos e instrumentos de medición. En tercer lugar, destaca la presentación, el análisis y la interpretación de los resultados. Si el estudio fue cuantitativo, se muestran los datos a través de tablas, gráficos, dibujos y figuras numeradas. Si la metodología fue cualitativa, los resultados se presentan como afirmaciones que resumen la información. En cuarto lugar están las conclusiones, en las que se retoman los objetivos y las hipótesis con la finalidad de responderlos teniendo en cuenta los resultados. También se pueden proyectar aspectos que no se han abordado y que pueden considerarse para futuras investigaciones. Por último, la presentación de la bibliografía y las fuentes. Se trata del listado bibliográfico que se ha citado en el cuerpo del trabajo, y en algunas ocasiones también se presenta la lista de fuentes consultadas. 
Este género ha sido abordado también por Castro Azuara y Sánchez Camargo (2015) quienes consideran que en el ámbito universitario mexicano la producción de tesinas de grado está decreciendo, y destacan la importancia y la utilidad que tiene la instancia de escritura de un trabajo de estas características para terminar los estudios de grado: tiene potencial epistémico vinculado al desarrollo de habilidades expositivas y argumentativas. Retoman la idea de movimientos retóricos de Swales (1990), Bhatia (1993) y Parodi (2008) para analizar una muestra de sesenta y tres tesinas de cuatro carreras de Humanidades a fin de descubrir su estructura. A partir del análisis, descubren la existencia de dos tipos de tesinas: Por un lado, estudios teóricos combinados con aplicaciones prácticas y, por otro, tesis de investigación-acción, que son mayoritarias en su muestra. A partir de estos datos, los autores decidieron hacer una descripción solo de las tesis abocadas a la investigación-acción. En cuanto a su organización retórica, destacan los agradecimientos, los índices, la introducción, capítulo o capítulos dedicados al desarrollo del planteamiento teórico, capítulo o capítulos dedicados al planteamiento metodológico, conclusión y referencias. Dentro de cada uno de estos segmentos textuales, identifican una serie de pasos estratégicos. Dentro de la introducción, destacan la presentación general del tema, la presentación del estado del conocimiento, la exposición de una tesis o problema, la exposición del objetivo o propósito, y un adelanto de la organización del trabajo. En el capítulo o los capítulos abocados al desarrollo teórico, se presentan los pasos estratégicos de identificación de la problemática, la 
presentación del estado de la cuestión, una síntesis de los planteamientos de uno o varios autores, destacando su valor. En el capítulo o los capítulos abocados al desarrollo metodológico se puede encontrar el planteamiento del problema y las preguntas que guían la investigación, el tipo de investigación y diseño de la misma, descripción de la población o muestra, las técnicas o instrumentos de recolección de datos y las unidades de análisis. En la conclusión recalcan la presencia de la exposición de la idea principal, el resumen de los resultados, el resumen del texto, la evaluación de ideas o resultados y sugerencias de investigaciones futuras derivadas de sus hallazgos. En este caso, nos llama la atención que no figure un capítulo específico para la exposición de resultados, teniendo en cuenta que sí destacan que en las conclusiones existe el resumen de esos resultados.

La estructura retórica que tomamos para nuestro análisis está derivada de la estructura prototípica del artículo científico y toma los pasos retóricos de Swales (1990). El modelo teórico será explicitado en el próximo apartado. Hemos decidido tomar este modelo teórico porque a partir de un estudio previo (Hael, 2010), hemos podido comprobar que las tesinas de grado de disciplinas de las Ciencias Sociales y Humanas se ajustan en general a esta estructura -a excepción del apartado discusión-, pero las tesinas de las Ciencias Naturales se ajustan en su totalidad: se respeta la estructura del artículo científico, con la diferencia de que el planteo del problema es más amplio que en un artículo de investigación, al igual que su desarrollo y su extensión, pero las partes prototípicas y los pasos retóricos se presentan de igual manera. Como nuestro estudio doctoral 
abarca seis disciplinas - dos de Ciencias Sociales y Humanas, dos de Ciencias Naturales y dos de Ciencias Exactas- hemos decidido aplicar este modelo basándonos en esos resultados anteriores. Además, como puede verse, los estudios referidos en este segmento dan cuenta de estructuras y pasos estratégicos muy similares, salvo en el caso del apartado discusión, que en ningún caso se presenta.

Existe otro antecedente vinculado a nuestro estudio que estudia la presencia de los pasos retóricos y de las partes prototípicas de los escritos académicos en ponencias elaboradas en el marco de un programa de investigaciónacción en una asignatura de primer año de la carrera de Letras de una universidad pública argentina (Padilla et al, 2010), que arroja como resultado que en la mayoría de las producciones, los estudiantes pudieron internalizar las partes y segmentos canónicos del discurso académico -a excepción del apartado discusión y de los pasos retóricos que se refieren a la ocupación del espacio de investigación, resumen de resultados propios y cuestiones abiertas-, luego de un proceso sostenido de tutoría académica. Además, encontraron que la mayoría de los estudiantes pudo armar su investigación a partir del planteo de hipótesis, confrontación de datos y conclusiones, lo que los acercaría a un modo de argumentación demostrativa.

\section{Marco teórico}

En este apartado expondremos con más detalle la clasificación propuesta por Gnutzmann y Oldenburg (1991) y Ciapuscio y Otañi (2002) para las partes y segmentos textuales 
del artículo científico, que según un estudio anterior (Hael, 2010) también puede ser considerado para las tesinas de licenciatura. Pondremos estas partes canónicas en vinculación con los pasos retóricos de Swales (1990) y los relacionaremos con tres formas de concebir la argumentación académica (Padilla et al, 2011).

Con respecto a las partes canónicas encontramos:

1) Una introducción, en la que se desarrollan partes como la presentación del problema, el estado de la cuestión, el lugar que ocupa la investigación dentro del campo disciplinar, el marco teórico, los objetivos y las hipótesis e interrogantes. La introducción plantea un punto argumentativo importante, que funciona a modo de premisas: los conceptos desarrollados por los teóricos de base de las investigaciones funcionan como punto de partida en el que los autores se apoyan. Así también, pueden surgir citas de autoridad, si se considera y se expresa que los postulados de los teóricos a los que se tiene en cuenta son acertados, mientras que pueden incorporarse citas polémicas, si se cree que los aportes teóricos realizados por otros autores no son suficientes y tienen limitaciones específicas. También puede darse a través de la mención de un marco teórico con el que abiertamente se encuentran en contra, pero al que no pueden desconocer en virtud de los antecedentes en relación con el tema. Recordemos que las premisas, las citas de autoridad y las citas polémicas son elementos básicos de la argumentación (Perelman y OlbrechtsTyteca, 1989), no solo académica, sino también de la argumentación cotidiana. Así también presenta otro punto significativo: las hipótesis e interrogantes y objetivos, porque 
ellos guiarán la realización de todo el trabajo, y el propósito fundamental del autor será probar que su hipótesis es acertada y hacer que los lectores adhieran a su modo de interpretar los datos (Padilla, 2005).

2) Una metodología de trabajo, en la que se explicita el corpus, la población, los materiales y los métodos empleados para la elaboración del trabajo de investigación. La metodología presenta otro elemento argumentativo importante, ya que en la descripción de cómo se ha realizado la investigación, se prueba que el procedimiento ha sido correcto en función de los objetivos planteados. En una buena argumentación académica, la metodología será coherente con los propósitos de la investigación.

3) Los resultados, donde el investigador muestra datos cualitativos, cuantitativos o ambos tipos de datos, que son los que comprueban la o las hipótesis propuestas, e intentan cumplir los objetivos abordados. Esta sección tiene, también, un componente argumentativo a resaltar porque el autor prueba mediante lugares de cantidad (datos cuantitativos) y lugares de calidad (datos cualitativos) -fundamentales en las teorías de la argumentación- lo que ha propuesto en sus hipótesis.

4) La discusión pone en relación los datos de la propia investigación con los de otras investigaciones sobre el tema emprendido; es un diálogo con lo que otros estudiosos pueden haber encontrado sobre el mismo problema de investigación. Es aquí donde la argumentación cobra un papel fundamental en cuanto a su componente polémico. Si bien algunas veces los 
resultados son similares, otras, disienten, de modo que se genera un diálogo entre la comunidad científica respecto del tema en cuestión.

5) En la conclusión aparecen pasos retóricos tales como el resumen de los resultados de la investigación, los puntos fuertes y débiles de la misma, $y$, por último, cuestiones abiertas a futuros estudios. En las conclusiones se retoma todo lo desarrollado con anterioridad y se destaca cómo han sido comprobadas las hipótesis principales o cómo han sido respondidos los interrogantes planteados, entre otras cuestiones de importancia.

Padilla et al (2011) vinculan las partes canónicas del artículo científico con tres posibilidades de argumentación: Una argumentación demostrativa que implica una articulación entre el marco teórico las hipótesis y/o preguntas de investigación, los datos y las conclusiones; Una argumentación persuasiva que busca que el modo de comunicar los resultados de la investigación sea el más eficaz, teniendo en cuenta a los posibles lectores; y por último, una argumentación dialéctica que se abre a la consideración de otros puntos de vista, y contrapone los resultados propios con los de investigaciones previas acerca del tema en cuestión.

\section{Metodología}

Para realizar esta investigación, tomamos un corpus de diez trabajos finales de grado de Ingeniería Civil de la biblioteca de una universidad pública argentina, unidad académica seleccionada para este estudio. Siguiendo a Hernández 
Sampieri, Fernández Collado y Baptista Lucio (2006), el acopio se realizó al azar a través del muestreo probabilístico aleatorio simple, sin previa lectura y selección del material puesto que, de este modo, consideramos que este resulta más representativo del modo en el que se construye el conocimiento en la disciplina abordada. El criterio empleado consistió en consultar el listado de trabajos finales de Ingeniería Civil presentados desde el año 2003 hasta 2012; a partir de esa consulta, se hizo un listado de todos los trabajos presentados por cada año. Una vez realizadas las listas, se seleccionó a partir de un sorteo, obteniendo un trabajo de cada año. Para preservar el anonimato (Meo, 2010), clave de la ética en investigación en Ciencias Sociales y Humanas, no hemos incluido en el cuerpo del artículo el listado de trabajos que conforman nuestro corpus.

Analizamos este corpus teniendo en cuenta las partes canónicas de producciones científicas propuestas por Gnutzmann y Oldenburg (1991) y Ciapuscio y Otañi (2002) y los pasos retóricos de Swales (1990), desarrollados en el marco teórico. A partir de estas categorías, elaboramos un cuadro en el que se explicita si cada trabajo presenta o no las partes canónicas y los pasos retóricos, para luego cuantificarlas y obtener porcentajes. Asimismo, extraeremos algunos ejemplos del corpus, que permitirán mostrar el modo en que los autores incluyen las categorías propuestas. En las citas haremos referencia a los trabajos finales como T. F. y su respectiva numeración, que es la misma que se presenta en la tabla porcentual. 
Si bien el diseño de investigación es mixto (porque se proponen técnicas tanto cualitativas como cuantitativas), en este artículo en particular, no se hará un análisis cualitativo profundo, puesto que preferimos priorizar lo porcentual. Asimismo, los ejemplos que se ofrecen conllevan un breve análisis, que será ahondado en futuras investigaciones.

\section{Resultados}

Luego de haber recapitulado brevemente acerca de los pasos retóricos y de las partes canónicas de los escritos académicos y científicos, nos abocaremos al análisis de los mismos en los trabajos finales de Ingeniería Civil que conforman nuestro corpus.

En el siguiente cuadro podrá observarse la presencia de las partes canónicas ya mencionadas, en las diez producciones estudiadas. 


\begin{tabular}{|c|c|c|c|c|c|c|c|c|c|c|c|}
\hline & $\mathrm{T} 1$ & $\mathrm{~T} 2$ & T3 & $\mathrm{T} 4$ & T5 & & $\mathrm{T} 7$ & T8 & & $\mathrm{T} 10$ & Total \\
\hline INTRODUCCIÓN & $\mathrm{X}$ & $\mathrm{X}$ & $\mathrm{X}$ & $\mathrm{X}$ & $\mathrm{X}$ & $\mathrm{X}$ & $\mathrm{X}$ & $\mathrm{X}$ & $\mathrm{X}$ & $\mathrm{X}$ & $\begin{array}{l}10 \text { de } 10 \\
=100 \%\end{array}$ \\
\hline $\begin{array}{l}\text { Presentación del } \\
\text { problema }\end{array}$ & $\mathrm{x}$ & $\mathrm{X}$ & $\mathrm{X}$ & $\mathrm{X}$ & $\mathrm{X}$ & $\mathrm{X}$ & $\mathrm{X}$ & $\mathrm{X}$ & $\mathrm{X}$ & $\mathrm{X}$ & $\begin{array}{l}10 \text { de } 10 \\
=100 \%\end{array}$ \\
\hline $\begin{array}{l}\text { Estado de la } \\
\text { cuestión }\end{array}$ & $\mathrm{x}$ & $\mathrm{x}$ & $\mathrm{x}$ & $\mathrm{x}$ & $\mathrm{x}$ & - & - & - & $\mathrm{x}$ & $\mathrm{x}$ & $\begin{array}{l}7 \text { de } 10 \\
=70 \%\end{array}$ \\
\hline $\begin{array}{l}\text { Lugar que ocupa la } \\
\text { investigación }\end{array}$ & $\mathrm{x}$ & $\mathrm{x}$ & $\mathrm{x}$ & $\mathrm{x}$ & $\mathrm{x}$ & - & - & $\mathrm{x}$ & - & $\mathrm{x}$ & $\begin{array}{l}7 \text { de } 10 \\
=70 \%\end{array}$ \\
\hline Marco teórico & $\mathrm{X}$ & $\mathrm{X}$ & $\mathrm{x}$ & $\mathrm{x}$ & $\mathrm{x}$ & $\mathrm{X}$ & - & - & $\mathrm{x}$ & $\mathrm{x}$ & $\begin{array}{l}8 \text { de } 10 \\
=80 \%\end{array}$ \\
\hline $\begin{array}{l}\text { Objetivos. } \\
\text { Propósitos }\end{array}$ & $\mathrm{X}$ & $\mathrm{x}$ & $\mathrm{x}$ & $\mathrm{x}$ & $\mathrm{x}$ & $\mathrm{x}$ & $\mathrm{x}$ & $\mathrm{X}$ & $\mathrm{x}$ & $\mathrm{x}$ & $\begin{array}{l}10 \text { de } 10 \\
=100 \%\end{array}$ \\
\hline $\begin{array}{l}\text { Hipótesis/ } \\
\text { preguntas } \\
\text { investigación }\end{array}$ & - & - & - & - & - & - & - & - & - & - & $\begin{array}{l}0 \text { de } 10 \\
=0 \%\end{array}$ \\
\hline ME TODOLOGIA & - & - & $\mathrm{X}$ & $\mathrm{X}$ & - & - & - & $\mathrm{X}$ & - & $\mathrm{X}$ & $\begin{array}{l}4 \text { de } 10 \\
=40 \%\end{array}$ \\
\hline Poblac & $\mathrm{X}$ & $\mathrm{X}$ & $\mathrm{X}$ & $\mathrm{X}$ & - & $\mathrm{X}$ & $\mathrm{X}$ & $\mathrm{X}$ & $\mathrm{X}$ & $\mathrm{X}$ & $\begin{array}{l}9 \text { de } 10 \\
=90 \%\end{array}$ \\
\hline Mater & $\mathrm{x}$ & $\mathrm{x}$ & $\mathrm{x}$ & $\mathrm{x}$ & $\mathrm{x}$ & $\mathrm{x}$ & $\mathrm{x}$ & $\mathrm{X}$ & $\mathrm{X}$ & $\mathrm{x}$ & $\begin{array}{l}10 \text { de } 10 \\
=100 \%\end{array}$ \\
\hline Métodos & $\mathrm{x}$ & $\mathrm{X}$ & $\mathrm{x}$ & $\mathrm{x}$ & $\mathrm{x}$ & $\mathrm{x}$ & $\mathrm{x}$ & $\mathrm{X}$ & $\mathrm{X}$ & $\mathrm{x}$ & $\begin{array}{l}10 \text { de } 10 \\
=100 \%\end{array}$ \\
\hline RESULTADOS & $\mathrm{x}$ & $\mathrm{X}$ & $\mathrm{x}$ & $\mathrm{x}$ & - & - & - & $\mathrm{X}$ & - & $\mathrm{x}$ & $\begin{array}{l}6 \text { de } 10 \\
=60 \%\end{array}$ \\
\hline Datos cuantitativos & $\mathrm{x}$ & $\mathrm{X}$ & $\mathrm{x}$ & $\mathrm{x}$ & $\mathrm{x}$ & $\mathrm{x}$ & $\mathrm{x}$ & $\mathrm{X}$ & $\mathrm{X}$ & $\mathrm{x}$ & $\begin{array}{l}10 \text { de } 10 \\
=100 \%\end{array}$ \\
\hline Datos cualitativos & - & $\mathrm{X}$ & - & - & - & - & - & - & - & - & $\begin{array}{l}1 \text { de } 10 \\
=10 \%\end{array}$ \\
\hline DISCUSION & - & 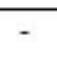 & & - & 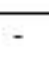 & - & - & $\mathrm{X}$ & & - & $1 \mathrm{de} 10$ \\
\hline
\end{tabular}


Como se puede observar en el cuadro, los trabajos presentan, a grandes rasgos, la estructura canónica de un artículo científico. Todos ellos $(100 \%)$ constan de una introducción, en la que presentan claramente el problema, y en la que plantean objetivos y propósitos concretos, que cumplirán en el desarrollo de su trabajo. Estas categorías cuentan con un porcentaje del 100\%. El marco teórico cuenta con un porcentaje del $80 \%$, y los antecedentes y el lugar que ocupa la investigación, como puede observarse, cuentan con un porcentaje del $70 \%$ en cada caso. Nos parece notorio que las preguntas de investigación o hipótesis no tengan cabida en ningún caso, por lo que podría tratarse de una recurrencia disciplinar. El $90 \%$ incluye el lugar que ocupa la investigación. Este hecho podría vincular la forma de concebir el quehacer científico en esta disciplina de manera más bien descriptiva. A modo de ilustración, podemos ver los siguientes ejemplos de las categorías más recurrentes:

En estos últimos años se ha considerado de importancia la recuperación de los valores naturales del río, entre otras cosas si es posible conseguir que un río vuelva a tener espacios de valor natural o recreativo, en este sentido significa lograr un encauzamiento con un enfoque respetuoso con el medio natural. No obstante hay que reconocer que un encauzamiento casi siempre supone una alteración o transformación del medio que en ocasiones puede ser importante (T. F. 4, p. 2).

Aquí vemos cómo presenta el problema a través de una reflexión sobre lo que ocurre, actualmente, con respecto a los encauces fluviales, para luego ofrecer objetivos acordes y 
resaltar, en virtud de esto mismo, el lugar que ocupa su investigación en el área científica de la Ingeniería Civil.

En cuanto a la definición de objetivos, podemos ver, en el T. F. 4, p. 1: "Se propone en este trabajo construir el modelo físico y realizar ensayos para estudiar la resistencia a la erosión de un suelo con cobertura vegetal”.

Esto ha motivado que en el campo de la ingeniería haya tenido especial interés el empleo de protecciones naturales que involucren cubiertas de arcillas y/o de pasto. En la bibliografía específica sobre el tema, no se cuenta con demasiada información sobre la resistencia que tienen estos tipos de protecciones a la acción de las corrientes. Por ello la necesidad de encarar estudios en modelo físico escala 1:1 o en prototipo donde se analicen la influencia de los distintos parámetros que intervienen en los procesos erosivos y resistentes y que no pueden ser tenidos en cuenta al trabajar en modelos a escala tales como la cohesión del suelo" (T.F. 4, p. 2).

En este fragmento, podemos observar cómo establece su nicho, el lugar que ocupa su investigación en el campo disciplinar en el que se desarrolla.

Como hemos mencionado con anterioridad, ninguno de los trabajos finales de esta disciplina presenta hipótesis ni preguntas de investigación.

En cuanto al marco teórico y los antecedentes, podemos mostrar los siguientes ejemplos:

Por otra parte, diversos estudios en este campo realizados en otros países determinaron curvas de 
velocidades críticas de erosión en función de su tiempo de permanencia del flujo donde se aprecia que mientras mayor es la duración de la velocidad actuante del flujo, el valor de la velocidad crítica tiende a reducirse. Así se determinaron valores de velocidades críticas para duraciones de hasta 50 horas pero esta condición en algunos casos puede resultar escasa (T. F. 4, p. 2).

En este ejemplo vemos la inclusión de un antecedente de investigación relacionado con el tema de su estudio, aunque no consigna las fuentes. En el marco teórico se dedica, sobre todo, a la definición y explicación del modelo físico y de cuestiones vinculadas a este, por ejemplo:

Modelos de validación: Son aquellos modelos empleados para verificar o extender los datos obtenidos de modelos numéricos. Como su objetivo es reproducir las condiciones asumidas en el modelo numérico, no se parecen en general al prototipo; Modelos de diseño: Tienen como objetivo tratar de predecir el comportamiento del prototipo, reproduciendo lo mejor posible todas las características y fuerzas presentes en el mismo. Este tipo de modelos intentan proveer una solución ingenieril a un problema. Su ejecución es complicada debido a los problemas de escala, obtención de los datos del prototipo para reproducirlos en el modelo y luego verificar la correcta respuesta del modelo; Modelos de procesos: Este tipo de modelo está diseñado para estudiar un proceso físico para desarrollar una nueva interpretación acerca del mismo (T. F. 4, p. 4).

En este ejemplo, podemos observar una clasificación acerca de los modelos físicos que ofrece el autor del trabajo final seleccionado para los ejemplos. A partir de esto, luego, en los 
resultados, expondrá los datos obtenidos del modelo físico escogido para su experimento.

La sección metodología cuenta con una frecuencia de aparición del $40 \%$ en el corpus analizado. Cabe aclarar que si bien en la mayoría de los textos no aparece como segmento textual, sí se cumplen los pasos retóricos. En el $60 \%$ en el que la metodología no es explicitada a través de un subtítulo, se la incluye mezclada con los resultados: se explica cómo se ha procedido y a continuación, se muestran los resultados obtenidos. Así es que el 100\% incluye los materiales y los métodos, y el $90 \%$, la población. En el ejemplo que sigue, del T. F. 4 (p. 10), se puede ver una breve descripción de materiales y métodos:

Los valores recuadrados de la izquierda corresponden a las alturas medidas en la regla del soporte del ADV y del molinete (figura 3.2). Las correspondientes a los puntos 1 a 8 se midieron utilizando la varilla de soporte fijada a $1,00 \mathrm{~m}$ del borde inferior de la mordaza del soporte, los puntos 9 a 16 se midieron con dicha varilla a 0,80 del mismo borde.

Se puede observar en la tabla en la que hemos cuantificado nuestras categorías de análisis, que el T. F. 4 sí presenta la metodología de manera explícita y los resultados por su lado. Es por eso que hemos ofrecido el ejemplo de ese caso. En el análisis de los resultados, también mostraremos un ejemplo de este mismo T. F. 4, pero también mostraremos un ejemplo de cómo se presenta la metodología y los resultados de manera conjunta en el T. F. 2, que es otro de nuestros seleccionados para ejemplificar ya que este modo de estructurar los trabajos 
es representativo de lo que ocurre en el corpus (recordemos que un $60 \%$ estructura presentando de manera mixta metodología y resultados).

En cuanto a los resultados, de manera similar a lo que ocurre con la metodología, solo el $60 \%$ de las tesis los presentan de manera explícita bajo un subtítulo con tal nombre. El 40\% restante expone los resultados en un apartado con un título cualquiera, en el que mezclan metodología y datos. Asimismo, todas las tesis (100\%) incorporan datos cuantitativos como forma probatoria de sus objetivos, por ejemplo, tomando el caso del T. F. 4, que separa metodología de resultados:

Las velocidades obtenidas con el ADV en dirección transversal al canal resultaron del orden de $1,5 \mathrm{~cm} / \mathrm{seg}$. que respecto a las obtenidas en dirección del escurrimiento, del orden de $45 \mathrm{~cm} / \mathrm{s}$, se consideraron como despreciables. Por lo tanto se analiza la distribución de velocidades en la sección en el sentido del escurrimiento únicamente (T. F. 4, p. 11).

Un 10\% incluye, además, datos cualitativos. Dentro de los datos cuantitativos, todos los tesistas apelan a fórmulas, gráficos, tablas y ecuaciones, lo que sería distintivo del modo en que se construye conocimiento en esta área. El trabajo que incluye, además, datos cuantitativos, recurre a citas de un reglamento acerca de la formación del hormigón (T. F. 2).

Un ejemplo de la exposición mixta de metodología y resultados, que podría ser característica de esta disciplina, puede ser encontrado, también, en el T. F. 2 (p. 45): 
Se realizó una distribución proporcional del coeficiente de balasto horizontal y vertical según las áreas de influencia de cada nodo componente de la malla. En la figura 3.3 se detalla la distribución de los 250 nodos y los 240 elementos de placa utilizados en la modelación.

En la figura 3.4 (por más detalles ver figura /A3/) se indican las cargas estáticas máximas consideradas en los puntos de contacto entre la placa de cimentación y el centro de mecanizado (...) El análisis de la tensión de contacto entre la fundación y el suelo de asiento se puede resumir con los siguientes resultados:^ Tensión de contacto máxima $=1.33 \mathrm{~kg} / \mathrm{cm} 2$; \& Tensión de contacto promedio $=0.31 \mathrm{~kg} / \mathrm{cm} 2 ;$ * Desplazamiento estático máximo $=0.6 \mathrm{~cm}$; \& Desplazamiento estático promedio $=0.5 \mathrm{~cm}$.

Vemos cómo, separados por unos pocos párrafos, el autor de este T. F. presenta la metodología empleada y los resultados obtenidos, y lo mismo ocurre con cada valor que calcula: explica la metodología de análisis y a continuación, los resultados obtenidos. Creemos que es una manera amable de presentar los resultados al virtual lector, puesto que exponer la metodología de cada cálculo y en un apartado alejado, exponer los resultados de los experimentos podría llevar a una mayor dificultad en la comprensión. En este sentido, tal vez podría vincularse esta manera de presentación mixta de metodología y resultados con una forma específica de argumentación retórica, que podría ser característica de esta disciplina: se emplearía esta forma mixta de presentación de metodología y resultados atendiendo al posible lector, para facilitar su comprensión y 
esto lo acerca a un tipo de argumentación retórica, diferente a la que se da en otras disciplinas, sobre todo de las Ciencias Sociales (Hael y Padilla, 2012).

El hecho de exponer datos cuantitativos en pos de cumplir con sus objetivos de investigación, de manera coherente con la metodología de trabajo propuesta, vincula a estos trabajos finales con la argumentación demostrativa (Padilla et al, 2011).

Ninguno de los tesistas incluye el apartado discusión en el cuerpo del trabajo, aunque el 50\% compara sus resultados con los de otras investigaciones, en la sección "resultados". Ante estos datos, encontramos que la argumentación dialéctica (Padilla et al, 2011) tiene cabida moderada en los trabajos analizados de esta disciplina. Podemos ejemplificar con un fragmento del T. F. 4, p. 60:

Se pensó en comenzar con una velocidad de $0.50 \mathrm{~m} / \mathrm{s}$ aun cuando según la bibliografía consultada [1], la misma no genera erosión independientemente del tiempo de permanencia, buscando de esta manera validar en cierta medida dicha recomendación. En correspondencia a esto se estableció para esta velocidad media de flujo una duración total de 60h, que supera en 10 horas el tiempo de permanencia en la bibliografía existente, como se muestra en la figura 4.11.

Discrepa con la bibliografía consultada en relación con la velocidad de inicio.

En lo que respecta a las conclusiones (90\%), uno de los pasos retóricos que suele aparecer dentro de este apartado final es el resumen de los resultados obtenidos en el cuerpo de la 
investigación, que en el caso de nuestro corpus cuenta con una frecuencia de aparición del $90 \%$. En cuanto a los puntos fuertes y los puntos débiles de la investigación, cuentan con una frecuencia del 50\%. Por último, el 40\% incorpora las cuestiones abiertas a futuras investigaciones.

A modo de ejemplo, en cuanto a resumen de resultados, podemos mostrar el siguiente caso:

Con la información obtenida de los ensayos realizados se caracterizó el campo de velocidades medio producido a lo largo del canal para diferentes condiciones de flujo pero no permitieron una caracterización de la dinámica de la turbulencia en el tiempo (T. F. 4, p. 100).

En cuanto a cuestiones abiertas a futuras investigaciones:

Con la información obtenida de los ensayos realizados se caracterizó el campo de velocidades medio producido a lo largo del canal para diferentes condiciones de flujo pero no permitieron una caracterización de la dinámica de la turbulencia en el tiempo (T. F. 4, p. 100).

En relación con esto, destaca que los puntos fuertes son haber construido la base para los futuros experimentos, lo que hace que la investigación no esté acabada y, por ende, tenga sus limitaciones, cumpliendo con otro de los pasos retóricos de la conclusión, aunque de manera menos explícita. 


\section{Conclusión}

A partir del análisis realizado, podemos afirmar que los trabajos estudiados se estructuran, a grandes rasgos, según las partes canónicas del artículo científico e incluyen, en menor o mayor medida, los movimientos retóricos de Swales (1990). Además, nuestros hallazgos coinciden, en general, con la estructura textual y los pasos estratégicos de las tesinas de licenciatura propuestos por Tamola de Siegel (2007) y por Castro Azuara y Sánchez Camargo (2015).

En nuestro análisis, hemos descubierto que si bien los tesistas no incluyen explícitamente todos los segmentos (lo que ocurre, sobre todo, con la metodología, los resultados y la discusión), sí lo hacen implícitamente y combinando pasos retóricos propios de la metodología, con pasos retóricos de los resultados y de la discusión, bajo un mismo subtítulo abarcativo. Esto podría tratarse de una regularidad disciplinar en el género analizado. Consideramos que esta manera de presentar los datos puede vincularse con un tipo específico de argumentación retórica (Padilla et al, 2010), que sería propio de los trabajos finales de Ingeniería Civil: presentar de manera conjunta metodología, resultados y discusión, facilita, en estos casos, la comprensión del virtual lector, lo que sería mucho más dificultoso si se lo presentara respetando las partes prototípicas del artículo científico.

Otro punto notorio es el hecho de que no plantean hipótesis ni preguntas de investigación, sino que sus investigaciones se basan únicamente en el cumplimiento de objetivos. Esto vincularía al tipo de investigación que se realiza en trabajos 
finales de esta área disciplinar con un enfoque en el que prima la descripción. Se construye conocimiento disciplinar práctico para luego explicar y describir cómo se llegó a esos resultados y a esas conclusiones.

La primacía de datos cuantitativos dentro del segmento "resultados" (con el 100\%) contra los datos cualitativos que solo cuentan con un $10 \%$ de frecuencia, muestra una recurrencia disciplinar que podría ser característica de los trabajos finales de Ingeniería Civil, y vincula al corpus de esta disciplina con una metodología cuantitativa en la construcción de conocimiento científico.

Por su parte, hemos descubierto que la discusión tiene mediana cabida en estos trabajos finales de grado, por lo que existiría moderada conciencia acerca del diálogo científico: con esto, podemos afirmar que la argumentación dialéctica tiene cabida en los trabajos analizados.

El punto central de esta investigación ha sido dar cuenta de la frecuencia de uso de las partes canónicas y de los movimientos retóricos de producciones científicas dentro de diez trabajos finales de Ingeniería Civil. Hemos podido establecer algunas relaciones con los tres tipos de argumentación académica: la dialéctica, la retórica y la demostrativa. En futuros trabajos de investigación se prevé complementar los datos obtenidos en el análisis del corpus con entrevistas a estudiantes de esta disciplina que se encuentren en proceso de escritura del trabajo final de grado o bien, sean graduados recientes y hayan pasado por esta instancia. 
Además, se pretende realizar un análisis cualitativo más profundo en relación con los ejemplos presentados. 


\section{Bibliografía}

Arnoux, E. \& M. Alvarado (1999). El apunte: Restricciones genéricas y operaciones de reformulación. M. C. Martínez (Ed.). Comprensión y producción de textos académicos expositivos y argumentativos. Cali: Cátedra UNESCO/Universidad del Valle, pp. 57-82.

Arnoux, E., Alvarado, M., Balmayor, E., Di Stefano, M., Pereira, M. \& Silvestri, A. (1996). El aprendizaje de la escritura en el ciclo superior. Z. Solana (Comp.), Adquisición de la escritura. Rosario: Ediciones Juglaría, pp. 199-234.

Arnoux, E., Di Stefano, M., Pereira, C. (2002). La lectura y la escritura en la universidad. Buenos aires: EUDEBA.

Bhatia, V. K. (1993). Analysing Genre: Language Use in Professional Settings. London: Longman.

Carlino, P. (2004). Escribir y leer en la universidad: responsabilidad compartida entre alumnos, docentes e instituciones. Textos en contexto. Leer y escribir en la universidad, 6, 5-21.

Carlino, P. (2005). Escribir, leer y aprender en la universidad. Una introducción a la alfabetización académica. Buenos Aires: Fondo de Cultura Económica.

Carlino, P. (2006). Concepciones y formas de enseñar escritura académica. Un estudio contrastivo. Signo y Seña. Revista del Instituto de Lingüística: "Procesos y prácticas de escritura en la educación superior”, 16, 71-116.

Carlino, P. (2011). Leer y escribir en las Ciencias Sociales en universidades argentinas. Contextos de Educación [artículo de investigación]. Recuperado de: http://www.hum.unrc.edu.ar/publicaciones/contextos/articu los/2011/pdfs/o1-carlino.pdf 
Carlino, P. (2013). Alfabetización académica diez años después. Revista Mexicana de Investigación Educativa, 18, (57), 355381.

Castro Azuara, M. C. y M. Sánchez Camago (2015). "Escribir en la universidad: la organización retórica del género tesina en el área de humanidades". Perfiles Educativos 148 (37), 50-67.

Ciapuscio, G. e I. Otañi (2002). Las conclusiones de los artículos de investigación desde una perspectiva contrastiva alemánespañol. Revista del Instituto de Investigaciones Lingüísticas y Literarias (RILL), 15, 117-133.

Gnutzmann, C. \& Oldenburg, H. (1991). Contrastive Text Linguistics in LSP-Research: Theoretical Considerations and some Preliminary Findings. Schröder, H. Subject-oriented Texts: Language for Special Purposes and Text Theory. New York/Berlin: Walter de Gruyter, pp. 101-136

Hael, M. V. (2010). Argumentación y exposición en escritos de estudiantes universitarios de diferentes disciplinas: los casos de Historia y Biología. San Miguel de Tucumán: Facultad de Filosofía y Letras, Universidad Nacional de Tucumán (en prensa).

Hael, M. V. y C. Padilla (2012). Citación en tesinas de Licenciatura en Ciencias de la Educación. Actas del VI Coloquio de Investigadores en Estudios del Discurso y III Jornadas Internacionales sobre Discurso e Interdisciplina, ALED-AR, Universidad Nacional de Quilmes, Ciudad de Bernal (en prensa).

Hernández Sampieri, R., C. Fernández Collado y P. Baptista Lucio (2006). Metodología de la Investigación. México D. F: Ed. Mc Graw Hill.

Hillard, V. y J. Harris, (2003). Making Writing Visible at Duke University. Peer Review, 15-17. 
Kelly, G. y C. Bazerman (2003). How Students Argue Scientific Claims: A Rhetorical-Semantic Analysis. Applied Linguistics (1-20). Oxford: Oxford Univ. Press.

Larrain, A. (2009). El rol de la argumentación en la alfabetización científica. Estudios Públicos, 116. Disponible en: www.cepchile.cl

Lea, M. y Street, B. V. (1998). Student Writing and Staff Feedback in Higher Education: An Academic Literacies Approach. Studies in Higher Education 23 (2), pp. 1-20.

Marin, M., Hall, B. (2003). Marcas de argumentatividad en los textos de estudio: obstáculo para lectores. En Actas del Congreso Internacional "La argumentación: Lingüística, Retórica, Lógica, Pedagogía”. Buenos Aires: EUDEBA, pp. 120

Marinkovich, J. y J. Salazar (2011). Representaciones sociales acerca del proceso de escritura académica: el caso de la tesis en una Licenciatura en Historia. Estudios pedagógicos, 1-20.

Marinkovich, J. y A. Córdova (2014). La escritura en la universidad: objeto de estudio, método y discursos. Revista Signos. Estudios de Lingüística, 40-63.

Meo, A. I. (2010). "Consentimiento informado, anonimato y confidencialidad en investigación social". Aposta: Revista de Ciencias Sociales $\mathrm{N}^{\mathrm{o}} 44$, pp. 1-30.

Motta-Roth, D. (2001). Redação acadêmica: princípios básicos. Santa Maria: Imprensa Universitária.

Motta-Roth, D. (2009). The role of context in academic text production and writing pedagogy. bazerman, C.; bonini, A.; figueiredo, D. (Eds.). Genre in a changing world. Fort Collins/Colorado/EUA: The WAC Clearinghouse (321-340) [Capítulo de libro]. Recuperado de: http://wac.colostate.edu/books/genre/chapter16.pdf. 
Padilla, C. (2005), Exposición y argumentación en el artículo científico. La escritura académica. Alfabetización y discurso científico. Tucumán: INSIL/UNT, pp. 57-78.

Padilla, C. y Carlino, P. (2010). Alfabetización académica e investigación acción: enseñar a elaborar ponencias en la clase universitaria. Alfabetización académica y profesional en el Siglo XXI: Leer y escribir desde las disciplinas. G. Parodi (Editor). Academia Chilena de la Lengua / Ariel. Santiago de Chile, pp. 153-182.

Padilla, C. et al (2010). La comunicación académica como construcción argumentativa: perspectivas de lectura y de escritura de estudiantes universitarios. Víctor M. Castel y Liliana Cubo de Severino (Eds.) La renovación de la palabra en el bicentenario de la Argentina. Los colores de la mirada lingüística. Mendoza: Editorial FFyL/UNCuyo.

Padilla, C. (2011). Yo argumento. Taller de prácticas de comprensión y producción de textos argumentativos. Córdoba: Comunicarte.

Parodi, G. (2008). La organización retórica del género Manual: ¿Una colonia encadenada? G. Parodi (Ed.). Géneros Académicos y Géneros Profesionales: Accesos discursivos para saber y hacer. Valparaíso: Ediciones Universitarias de Valparaíso.

Parodi, G. (2010). Alfabetización académica y profesional en el Siglo XXI: Leer y escribir desde las disciplinas. Santiago de Chile: Academia Chilena de la Lengua/Ariel.

Perelman, Ch. y L. Olbrechts-Tyteca (1989). Tratado de la Argumentación. La nueva retórica. Traducción de J. Sevilla Muñoz. Madrid: Gredos.

Perin, D., A. Keselman y M. Monopoli (2003). The academic writing of community college remedial students: text and learner variables. Higher Education (1-20), $\mathrm{N}^{\mathrm{O}}$ 45, Netherlands: Kluwer Academic Publishers. 
Soto Vergara, G. (2002). La argumentación en artículos científicos escritos en español. Actas del Congreso Internacional La Argumentación: Lingüística, Retórica, Lógica, Pedagogía, Universidad de Buenos Aires, Buenos Aires.

Soto Vergara, G. (2004-2005). La estructuración jerárquica de la información en el discurso escrito: segmento de orientación y núcleo informativo. Lenguas Modernas, $\mathrm{N}^{\mathrm{O}} 30$, pp. 7-24.

Soto Vergara, G. (2005). Las construcciones de agente degradado en la sección 'método' de los artículos científicos. Anamaria Harvey (Comp.).En torno al discurso. Contribuciones de América Latina. Ediciones Universidad Católica de Chile: Chile, pp. 111-125.

Soto Vergara, G., R. Martínez y S. Sadowsky (2005). Verbos y sustantivos en textos científicos. Análisis de variación en un corpus de textos de ciencias aplicadas, naturales, sociales y humanidades. Philologia Hispalensis (Sevilla), XIX, pp. 169187.

Soto Vergara, G. y C. Zenteno (2001-2003). La subtopicalización en el discurso científico escrito. Lenguas Modernas $\mathrm{N}^{\circ}$ 28-29, pp. 29-52.

Soto Vergara, G. y C. Zenteno (2004). Los sintagmas nominales en textos científicos escritos en español. Estudios de Lingüística, Universidad de Alicante, España, pp. 275-292.

Swales, J. (1990). Genre Analysis. English in Academic and Research Settings. Cambridge, Cambridge University Pres.

Tamola de Spiegel, D. (2007). La tesina de licenciatura. Cubo de Severino, L. (Coord.). Los textos de la ciencia. Principales clases del discurso académico-científico. Córdoba (Argentina), Comunicarte Editorial, pp.235-263.

Tapia, M. y J. Marinkovich (2013). Representaciones sociales sobre la escritura de la tesis en carreras del área de 
Humanidades: Periodismo y Trabajo Social. RBLA, Belo Horizonte, vol. 13, $\mathrm{N}^{0}$ 1, pp. 145-169.

Vázquez, A. y M. Miras (2004). Cómo se representan estudiantes universitarios las tareas de escritura. Reunión Internacional "Mente y Cultura: Cambios representacionales en el aprendizaje”. Bariloche, UNComahue.

Velásquez Rivera, M. y Córdova Jiménez, A (2011). Representaciones Sociales acerca de la Escritura Académica en un Programa de Licenciatura en Arte. Actas de las Jornadas Nacionales Cátedra UNESCO de Lectura y Escritura: "Lectura, escritura y aprendizaje disciplinar", Facultad de Ciencias Humanas, Universidad Nacional de Río Cuarto.

Wolfe, Ch. (2011). Argumentation Across the Curriculum. Written Comunication. $\mathrm{N}^{\mathrm{O}}$ 28. SAGE.

Zuber-Skerritt, O. y Ch. Perry (2002). Action research within organizations and university thesis writing. The Learning Organisation. Vol. 9, $\mathrm{N}^{\circ}$ 4, MBC UP Limited.

Fecha de recepción: 27/05/2015

Fecha de aprobación: 27/08/2015 